\title{
Delivery of Adjuvant Oxaliplatin for Colon Cancer: Insights From Routine Clinical Practice
}

Natasha Satkunam, MBBS, MSc $c^{\mathrm{a}}$; Xuejiao Wei, MSc $c^{\mathrm{a}}$ J James J. Biagi, MD, MASc ${ }^{\mathrm{b}}$; Sulaiman Nanji, MD, PhD ${ }^{\mathrm{b}, \mathrm{c}}$; and Christopher M. Booth, MD

\section{Abstract}

Background: Adjuvant oxaliplatin is now a standard treatment option for patients with early-stage colon cancer. However, treatment delivery and outcomes achieved in routine practice are not well described. Methods: All cases of colon cancer diagnosed in Ontario from 2002 to 2008 were identified using the Ontario Cancer Registry. Pathology reports were obtained for a $25 \%$ random sample to identify stage II and III cases; patients treated with adjuvant oxaliplatin were included in this analysis. Treatment records were reviewed to identify oxaliplatin dose reductions or omissions. Modified Poisson regression was used to evaluate factors associated with dose reduction/omission. Cox proportional hazards model was used to explore factors associated with cancer-specific survival (CSS) and overall survival (OS). Results: The study population included 532 patients; 88\% (469/532) had stage III disease. The mean/median number of oxaliplatin cycles delivered was $10 / 12$. A dose reduction/omission of oxaliplatin occurred in $54 \%$ of cases (288/532), and the dose was subsequently escalated in $34 \%$ of these (97/288). Women were more likely than men to have dose reduction/omission (relative risk, 1.29; 95\% $\mathrm{Cl}, 1.10-1.51$ ). Dose reduction/ omission was not associated with inferior CSS (hazard ratio [HR], 0.76; 95\% Cl, 0.51-1.14) or OS (HR, 0.81; 95\% Cl, 0.59-1.13). Five-year CSS and OS of all cases were $77 \%(95 \% \mathrm{Cl}, 72-81)$ and $72 \%(95 \% \mathrm{Cl}, 68-76)$, respectively. On-treatment mortality rates were $1 \%$ and $3 \%$ within 30 and 90 days of oxaliplatin, respectively. Conclusions: Dose reductions of adjuvant oxaliplatin are common in routine practice but are not associated with inferior survival. Long-term survival achieved in the general population is comparable to the results of clinical trials.

J Natl Compr Canc Netw 2016;14(12):1548-1554

\section{Background}

Adjuvant chemotherapy (ACT) with 5-fluorouracil (FU) has been the standard of care for stage III or highrisk stage II colon cancer since the 1990s. ${ }^{1}$ This treatment is based on a series of randomized clinical trials (RCTs) showing a $30 \%$ reduction in death, translating into an absolute improvement in 5-year overall survival (OS) of $10 \%$ to $25 \%$. $^{2}$ Oral capecitabine is known to offer com-

From the aDivision of Cancer Care and Epidemiology, Queen's University Cancer Research Institute, and Departments of 'Oncology, 'Surgery, and dPublic Health Sciences, Queen's University, Kingston, Canada.

Submitted June 21, 2016; accepted for publication September 7, 2016.

The authors have disclosed that they have no financial interests,

arrangements, affiliations, or commercial interests with the manufacturers of any products discussed in this article or their competitors. Dr. Booth is supported as a Canada Research Chair in Population Cancer Care. This work was also supported by the Canada Foundation for Innovation and the Canadian Institutes of Health Research.

Parts of this material are based on data and information provided by Cancer Care Ontario. However, the analysis, conclusions, opinions, and statements expressed herein are those of the authors and not necessarily those of Cancer Care Ontario. parable efficacy and is often substituted for intravenous 5-FU. ${ }^{3}$ In 2004, the pivotal MOSIAC trial demonstrated that the addition of oxaliplatin to 5-FU (FOLFOX) led to improved disease-free survival. ${ }^{4}$ Similar findings were observed in a second RCT. ${ }^{5}$ Updated analyses of the MOSAIC trial demonstrated oxaliplatin is associated with improved survival at 10 years $(72 \%$ vs $67 \% ; P=.043) .^{5}$ Accordingly, the standard of care for patients with stage
This study was supported by the Institute for Clinical Evaluative Sciences (ICES), which is funded by an annual grant from the Ontario Ministry of Health and Long-Term Care (MOHLTC). The opinions, results, and conclusions reported in this paper are those of the authors and are independent from the funding sources. No endorsement by ICES or the Ontario MOHLTC is intended or should be inferred.

Dr. Booth had full access to all the data in the study and takes responsibility for the integrity of the data and the accuracy of the data analysis.

Correspondence: Christopher M. Booth, MD, Division of Cancer Care and Epidemiology, Queen's University Cancer Research Institute, 10 Stuart Street, Kingston, ON, K7L 3N6, Canada. E-mail: boothc@kgh.kari.net 
III and high-risk stage II colon cancer includes ACT with 5-FU/capecitabine with or without oxaliplatin. ${ }^{6,7}$

It is well-known that efficacy observed in clinical trials may not translate into effectiveness in the general population. ${ }^{8,9}$ This may relate to suboptimal adoption of new therapies in clinical practice. It may also relate to the fact that patients, providers, and health systems differ considerably between high-volume specialized centers that conduct trials and routine practice. ${ }^{10}$ Population-based outcome studies are useful to describe practice and outcomes achieved in the real world. ${ }^{11,12}$ Although a number of studies have previously reported rates of ACT use in colon cancer in routine clinical practice, ${ }^{13-15}$ there is limited literature describing details of how oxaliplatin is delivered in routine practice.

Knowledge of oxaliplatin delivery in routine practice comes from a handful of small populationbased studies (sample size, 114-281 patients). ${ }^{16-18}$ From these studies it is clear that a substantial proportion of patients will have a dose reduction in oxaliplatin. Whether dose reductions of oxaliplatin compromise long-term outcomes remains unclear, with conflicting results reported by 2 small studies. ${ }^{17,18}$ OS at 5 years has been reported as $70 \%$ and $78 \%$, but these reports had 114 and 182 oxaliplatin cases, respectively. ${ }^{17,18}$ In a single study, mortality within 60 days of starting adjuvant oxaliplatin was reported as $1 \%$ to $2 \% .{ }^{19}$ In the pivotal MOSAIC trial, OS at 5 years was closer to $80 \%$ and treatmentrelated mortality was less than $1 \% .4,20$

Given the gaps in existing knowledge, we undertook a population-based study to describe the delivery of oxaliplatin ACT for colon cancer in Ontario. The study objectives were to (1) describe delivery of oxaliplatin in routine practice; (2) identify factors associated with dose reduction; and (3) explore survival outcomes associated with oxaliplatin-based ACT in routine practice and whether dose reductions are associated with inferior survival.

\section{Methods}

\section{Study Design and Population}

This is a secondary analysis of a population-based, retrospective cohort study to describe the management and outcome of resected stage II and III colon cancer in the Canadian province of Ontario. The primary results and study cohort were recently re- ported. ${ }^{21}$ Ontario has a population of approximately 13.5 million and a single-payer universal health insurance program. The study population included patients who underwent resection of stage II and III colon cancer in Ontario between 2002 and 2008. To identify the study cohort, we used the Ontario Cancer Registry (OCR) to identify all incident cases of colorectal cancer (CRC) in Ontario diagnosed in 2000 to 2008. The OCR does not capture disease stage for all patients; therefore, we obtained surgical pathology reports for a random sample of $25 \%$ of cases. Reports were not available for patients who underwent surgery in 2005; as such, the study cohort is restricted to patients who had surgery in 2002 to 2004 and 2006 to 2008. The study was approved by the Research Ethics Board of Queen's University.

\section{Data Sources and Linkage}

The OCR is a passive, population-based cancer registry that captures diagnostic and demographic information on at least $98 \%$ of all incident cases of cancer in the province of Ontario. ${ }^{22}$ The OCR also provides information about vital status and cause of death. Records of hospitalization from the Canadian Institute for Health Information (CIHI) provided information about surgical procedures; these records are known to have a very high level of completeness for CRC surgery. ${ }^{23}$ Provincial physician billing records from the Ontario Health Insurance Plan, treatment records from regional cancer centers, and provincial records of the New Drug Funding Program were used to identify chemotherapy use. A team of trained data abstractors reviewed the pathology reports and entered information about extent of disease into an electronic database.

\section{Measures and Outcomes}

Indicators of the socioeconomic status of the community in which patients resided at diagnosis were linked as described previously. ${ }^{24} \mathrm{Q} 1$ represents the communities where the poorest 20\% of the Ontario population resided. Geographic regions reflect the catchment areas for Ontario's regional cancer centers. Comorbidity was classified using the Charlson index modified for administrative data based on all noncancer diagnoses recorded during any hospital admission within 5 years before surgery. ${ }^{25}$

ACT was defined as chemotherapy initiated within 16 weeks after surgery. Drug delivery records 
Satkunam et al

were reviewed to identify cases treated with oxaliplatin. Cases also treated with 5 -FU were classified as FOLFOX and cases with associated records of capecitabine were classified as CAPOX. Because oxaliplatin was reimbursed by the New Drug Funding Program, there were some cases in which oxaliplatin was identified but the concomitant chemotherapy (ie, 5-FU or capecitabine) was not known. Oxaliplatin dose reduction was defined as the delivery of any dose of oxaliplatin that was less than the initial dose. Cases with a treatment record showing the delivery of a fluoropyrimidine without oxaliplatin were classified as having a dose omission.

Cancer-specific survival (CSS) and OS were determined from date of surgery. To account for possible cause-of-death miscoding, CSS included death from any cancer. Complete information about vital status in the OCR was available up to December 31, 2012; cause of death was available up to December 31, 2010.

\section{Statistical Analysis}

Comparisons of proportions between study groups were made using the chi-square test. Survival was determined from date of surgery using the KaplanMeier technique and comparisons between groups were made using the log-rank test. Factors associated with dose reduction/omission were evaluated using modified Poisson regression. The association between patient-, disease-, and treatment-related factors with OS/CSS was evaluated using the Cox proportional hazards regression model. Results were considered statistically significant at a $P$ value of less than 0.05. All analyses were performed using SAS 9.3 (SAS Institute Inc., Cary, NC).

\section{Results}

\section{Study Population}

Among the 5,519 patients with stage II and III colon cancer, $42 \%$ received ACT. A specific ACT regimen was identified for $67 \%$ cases; among these cases, oxaliplatin was delivered to $35 \%$. A total of 547 cases were treated with oxaliplatin within 16 weeks of surgery; $97 \%$ (532/547) had 12 or fewer cycles and 3\% $(15 / 547)$ had more than 12 cycles. Because it was not clear whether the cases with more than 12 cycles received ACT or palliative chemotherapy, they were excluded from the analysis. The study population therefore includes 532 cases. Characteristics of the study population are shown in Table 1 . The median age was 61 years and 54\% (289/532) were male; $88 \%$ (469/532) of cases had stage III disease. Among the 63 patients with stage II disease, 27\% (17/63) had T4 tumors, $13 \%(8 / 63)$ had fewer than 12 lymph nodes resected, 27\% (17/63) had lymphovascular invasion, and 21\% (13/63) had poorly differentiated histology. Among the 532 patients treated with oxaliplatin,

\begin{tabular}{|c|c|}
\hline \multicolumn{2}{|c|}{$\begin{array}{l}\text { Table 1. Characteristics of Patients With } \\
\text { Cancer Treated With Adjuvant } \\
\text { Chemotherapy }\end{array}$} \\
\hline & $\mathrm{N}(\%)$ \\
\hline \multicolumn{2}{|l|}{ Patient-related } \\
\hline \multicolumn{2}{|l|}{ Age, y } \\
\hline $20-49$ & $75(14 \%)$ \\
\hline $50-59$ & $147(28 \%)$ \\
\hline $60-69$ & $208(39 \%)$ \\
\hline$\geq 70$ & $102(19 \%)$ \\
\hline \multicolumn{2}{|l|}{ Sex } \\
\hline Male & $289(54 \%)$ \\
\hline Female & $243(46 \%)$ \\
\hline \multicolumn{2}{|l|}{ SES by quintile ${ }^{a}$} \\
\hline 1 & $90(17 \%)$ \\
\hline 2 & $111(21 \%)$ \\
\hline 3 & $128(24 \%)$ \\
\hline 4 & $90(17 \%)$ \\
\hline 5 & $113(21 \%)$ \\
\hline \multicolumn{2}{|l|}{ Charlson comorbidity score } \\
\hline 0 & $475(89 \%)$ \\
\hline 1 & $37(7 \%)$ \\
\hline$\geq 2$ & $20(4 \%)$ \\
\hline \multicolumn{2}{|l|}{ Disease-related } \\
\hline \multicolumn{2}{|l|}{ Histology, lymphovascular invasion } \\
\hline Yes & $249(47 \%)$ \\
\hline No & $264(50 \%)$ \\
\hline NA & $19(4 \%)$ \\
\hline \multicolumn{2}{|l|}{$\mathrm{N}$ stage } \\
\hline 0 & $63(12 \%)$ \\
\hline 1 & $251(47 \%)$ \\
\hline 2 & $218(41 \%)$ \\
\hline \multicolumn{2}{|l|}{ T stage } \\
\hline$\leq 1$ & $8(2 \%)$ \\
\hline 2 & $36(7 \%)$ \\
\hline 3 & $343(64 \%)$ \\
\hline 4 & $145(27 \%)$ \\
\hline \multicolumn{2}{|l|}{ Grade } \\
\hline Well to moderately differentiated & $419(79 \%)$ \\
\hline Poorly differentiated & $107(20 \%)$ \\
\hline Unstated & $6(1 \%)$ \\
\hline \multicolumn{2}{|l|}{ Concomitant chemotherapy } \\
\hline 5-FU (FOLFOX) & $397(75 \%)$ \\
\hline Capecitabine (CAPOX) & $26(5 \%)$ \\
\hline Unknown & $109(20 \%)$ \\
\hline
\end{tabular}

Abbreviations: NA, not applicable; SES, socioeconomic status. aQuintile 1 represents the communities where the poorest $20 \%$ of the Ontario population resided. 
we were able to identify concomitant use of intravenous 5 -FU in $75 \%(397 / 532)$ and concomitant capecitabine in $5 \%$ (26/532). The remaining 20\% of cases $(109 / 532)$ received oxaliplatin but we were unable to identify whether it was delivered with 5-FU or capecitabine.

\section{Chemotherapy Delivery}

The mean number of oxaliplatin cycles was 10 . Half of the cases $(269 / 532 ; 51 \%)$ received 12 cycles of oxaliplatin. Oxaliplatin dose was reduced or omitted in $54 \%$ of cases (288/532); in 34\% of these cases (97/288), oxaliplatin was subsequently reintroduced and/or dose-escalated.

Factors associated with dose reduction/omission are shown in Table 2. Age, comorbidity, and extent of disease were not associated with dose reduction/omission. However, women were substantially more likely than men to have a dose reduction/omission (61\% vs $48 \% ; P=.002)$; this persisted on multivariate analysis (relative risk [RR], 1.29; 95\% CI, 1.10-1.51).

\section{Outcomes}

Death rates within 30 and 90 days of any dose of oxaliplatin were $1 \%(7 / 532)$ and 3\% (16/532), respectively. The 5-year CSS and OS rates of all cases were $77 \%$ (95\% CI, 72-81) and 72\% (95\% CI, 68-76), respectively. As shown in Table 3, factors associated with inferior survival include advanced age, lymphovascular invasion, and poorly differentiated histology. Oxaliplatin dose reduction/omission was not associated with inferior CSS (hazard ratio [HR], 0.76; 95\% CI, 0.51-1.14) or OS (HR, 0.81; 95\% CI, 0.59-1.13).

\section{Discussion}

This population-based study describes the delivery of adjuvant oxaliplatin for stage II and III colon cancer in routine clinical practice. This is the largest such study in the literature and has several important findings. First, dose reduction/omission of oxaliplatin is common, occurring in approximately half of patients in routine practice. The dose will be subsequently increased in one-third of these cases. Second, we have found that dose reduction/omission is considerably more common in women compared with men. The reasons for this are not clear. Third, our data suggest that oxaliplatin dose reduction/omission is not associated with inferior long-term survival. Finally, long-

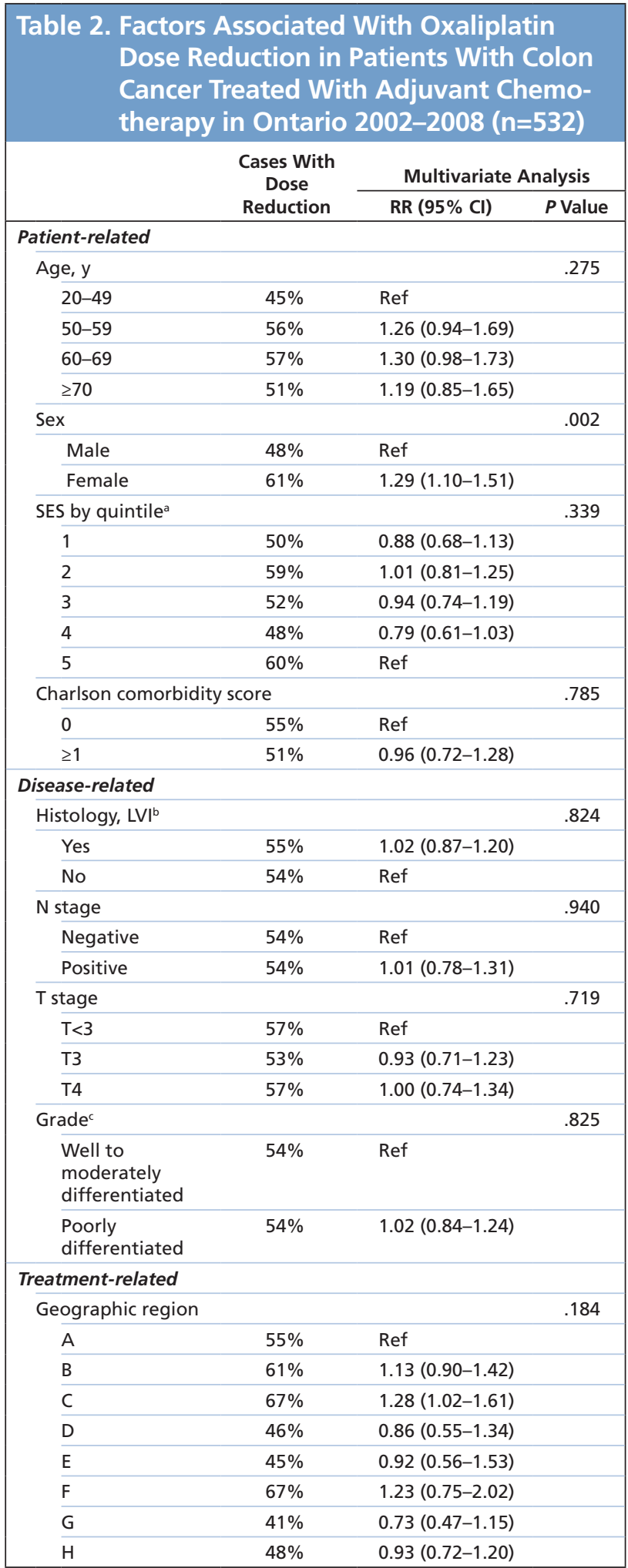

Abbreviations: LVI, lymphovascular invasion; Ref, referent; RR, relative risk; SES, socioeconomic status.

aQuintile 1 represents the communities where the poorest $20 \%$ of the Ontario population resided.

b19 patients with unstated LVI were excluded from the analysis.

${ }^{c}<6$ patients with unstated grade were excluded from the analysis. 
Satkunam et al

\begin{tabular}{|c|c|c|c|c|c|c|}
\hline \multirow[b]{2}{*}{ Characteristic } & \multicolumn{3}{|c|}{ CSS } & \multicolumn{3}{|c|}{ os } \\
\hline & 5-y CSS & $\begin{array}{c}\text { Multivariate } \\
\text { Analysis } \\
\text { HR }(95 \% \mathrm{Cl}) \\
\end{array}$ & $P$ Value & $5-y$ OS & $\begin{array}{c}\text { Multivariate } \\
\text { Analysis } \\
\text { HR }(95 \% \mathrm{Cl})\end{array}$ & $P$ Value \\
\hline \multicolumn{7}{|l|}{ Patient-related } \\
\hline Age, y & & & .003 & & & .002 \\
\hline $20-49(n=75)$ & $88 \%$ & Ref & & $82 \%$ & Ref & \\
\hline $50-59(n=147)$ & $77 \%$ & $1.79(0.79-4.09)$ & & $76 \%$ & $1.81(0.93-3.51)$ & \\
\hline $60-69(n=208)$ & $79 \%$ & $2.50(1.15-5.45)$ & & $71 \%$ & $2.31(1.23-4.33)$ & \\
\hline$\geq 70(n=102)$ & $59 \%$ & $4.19(1.83-9.60)$ & & $61 \%$ & $3.42(1.75-6.68)$ & \\
\hline Sex & & & .926 & & & .861 \\
\hline Male $(n=289)$ & $76 \%$ & Ref & & $73 \%$ & Ref & \\
\hline Female $(n=243)$ & $76 \%$ & $1.02(0.68-1.54)$ & & $71 \%$ & $0.97(0.70-1.35)$ & \\
\hline \multicolumn{2}{|c|}{ Charlson comorbidity score } & & .211 & & & .249 \\
\hline $0(n=475)$ & $78 \%$ & Ref & & $74 \%$ & Ref & \\
\hline $1+(n=57)$ & $N A^{a}$ & $1.43(0.82-2.51)$ & & $60 \%$ & $1.32(0.82-2.12)$ & \\
\hline \multicolumn{7}{|l|}{ Disease-related } \\
\hline T stage & & & .244 & & & .080 \\
\hline$<\mathrm{T} 3(\mathrm{n}=44)$ & $89 \%$ & Ref & & $86 \%$ & Ref & \\
\hline T3 $(n=343)$ & $78 \%$ & $2.44(0.76-7.87)$ & & $73 \%$ & $2.70(1.10-6.68)$ & \\
\hline $\mathrm{T} 4(\mathrm{n}=145)$ & $70 \%$ & $2.77(0.84-9.13)$ & & $66 \%$ & $2.89(1.14-7.30)$ & \\
\hline $\mathrm{N}$ stage & & & .078 & & & .687 \\
\hline Negative $(n=63)$ & $80 \%$ & Ref & & $78 \%$ & Ref & \\
\hline Positive $(n=469)$ & $76 \%$ & $0.57(0.31-1.06)$ & & $71 \%$ & $0.89(0.51-1.55)$ & \\
\hline $\mathrm{LVI}^{\mathrm{b}}$ & & & .001 & & & $<.001$ \\
\hline Yes $(n=249)$ & $67 \%$ & $2.08(1.32-3.27)$ & & $64 \%$ & $1.88(1.33-2.67)$ & \\
\hline No $(n=264)$ & $85 \%$ & Ref & & $80 \%$ & Ref & \\
\hline Grade $^{c}$ & & & $<.001$ & & & .010 \\
\hline $\begin{array}{l}\text { Well to moderately } \\
\text { differentiated } \\
(n=419)\end{array}$ & $81 \%$ & Ref & & $74 \%$ & Ref & \\
\hline $\begin{array}{l}\text { Poorly } \\
\text { differentiated } \\
(n=107)\end{array}$ & $61 \%$ & $2.58(1.67-4.00)$ & & $63 \%$ & $1.65(1.13-2.42)$ & \\
\hline Oxaliplatin dose reduct & ion & & .184 & & & .221 \\
\hline Yes $(n=288)$ & $76 \%$ & $0.76(0.51-1.14)$ & & $73 \%$ & $0.81(0.59-1.13)$ & \\
\hline No $(n=244)$ & $79 \%$ & Ref & & $71 \%$ & Ref & \\
\hline
\end{tabular}

Abbreviations: CSS, cancer-specific survival; HR, hazard ratio; LVI, lymphovascular invasion; NA, not applicable; OS, overall survival; Ref, referent a5-year CSS cannot be calculated for this subgroup due to the low number of CSS events.

b19 patients with unstated LVI were excluded from the analysis.

${ }^{c}<6$ patients with unstated grade were excluded from the analysis.

term survival of patients in routine practice is comparable to that seen in the relevant clinical trials.

It is worth considering the results of our study in light of the relevant clinical trials. The MOSAIC trial randomized 2,246 patients with stage II or III colon cancer to 12 cycles of FOLFOX or infusional 5 -FU; $75 \%$ of patients received 12 cycles of oxaliplatin. The initial report in 2004 demonstrated improved disease-free survival. ${ }^{4}$ Longer-term analyses confirmed that this translated into a clinically meaningful difference in OS. ${ }^{20}$ The NSABP CO7 trial has shown that FLOX (a slightly different oxaliplatinbased regimen) improved disease-free survival. ${ }^{5}$ An updated analysis in 2011 did not yet show evidence of improved OS. ${ }^{26}$

We are aware of several other reports that describe delivery of adjuvant oxaliplatin for colon cancer in routine practice. Sanoff et $\mathrm{al}^{14,19}$ published 2 studies using registry data from the United States. They found evidence of superior effectiveness of FOLFOX compared with 5-FU monotherapy among patients older than 75 years. ${ }^{14}$ They also found evi- 
dence of greater toxicity compared with 5-FU. ${ }^{19}$ These studies do not describe long-term survival or details related to dose reduction or number of cycles. The authors attributed deaths that occurred between 30 and 90 days after surgery to chemotherapy; $1 \%$ to $2 \%$ of cases died within this period.

We are aware of 3 studies that have described details of adjuvant oxaliplatin delivery in routine practice. Using a population-based registry in the Netherlands, van Gils et $\mathrm{a}^{16}$ described the delivery of ACT to 391 patients with stage III colon cancer; oxaliplatin was delivered in 281 cases. Details pertaining to dose intensity were available for 102 cases. The authors reported that the median number of cycles was 12 for FOLFOX and 7 for CAPOX. Fifty percent of cases received a full course of treatment. Up to $70 \%$ of patients required a dose reduction or interruption in the delivery of oxaliplatin. Our own results are consistent with these findings. Aspinall et $\mathrm{al}^{17}$ reported a similar study using data from Veteran Affairs hospitals in the United States. They identified 182 patients who received oxaliplatin. Consistent with the results from our study and that of van Gils et al, ${ }^{16} 53 \%$ of patients received a full course of oxaliplatin, and dose reductions were common. However, contrary to our findings, Aspinall et $\mathrm{al}^{17}$ found that dose reductions were associated with inferior OS and disease-free survival. In a third study, Smoragiewicz et $\mathrm{al}^{18}$ identified 114 patients with stage II or III colon cancer treated with FOLFOX in British Columbia, Canada. Their findings are consistent with ours: the median number of cycles completed was 12 , and $42 \%$ of cases received a dose reduction of oxaliplatin. In their analysis, dose reductions were not associated with inferior OS or relapse-free survival.

Our data provide insight into outcomes achieved with adjuvant oxaliplatin in routine clinical practice. Our observed rate of OS at 5 years $(72 \%)$ is comparable to rates seen in other population-based reports $(70 \% \text { and } 78 \%)^{17,18}$ and the MOSAIC trial (approximately 80\%). ${ }^{20}$ On-treatment mortality rates ( $1 \%$ within 30 days and $3 \%$ within 90 days) are also comparable to rates seen in the MOSAIC $(0.5 \%$ within 30 days of oxaliplatin) and NSABP CO7 (1.4\% within 60 days of oxaliplatin) clinical trials.,

One unexpected finding that emerged from our study was the substantially greater rate of dose reduction/omission in women compared with men. To our knowledge, this has not been previously reported in other trials of CRC. Our data do not provide insight into the causal mechanism for this observation. However, evidence suggests that women are more likely to develop 5-FU toxicity ${ }^{27,28}$ and oxaliplatin hypersensitivity. ${ }^{29}$

Although our study provides detailed data regarding delivery of adjuvant oxaliplatin in routine clinical practice, several methodologic limitations merit comment. The study population was identified using linked administrative health databases. Although the OCR and the CIHI data sets are known to be consistent and complete, ${ }^{22,23}$ it is possible that our results may be biased by misclassification. Although the electronic data sources used in this study describe general aspects of disease, treatment, and outcome for all patients in the province, detailed information related to carcinoembryonic antigen level, toxicity, reason for dose reduction/omission, and performance status is not available and limits our analysis. Importantly, body surface area was not available, and for this reason we were not able to determine relative dose intensity. The existing data sets did not allow us to identify whether oxaliplatin was delivered with intravenous 5 -FU or oral capecitabine in $20 \%$ of cases. Finally, as with any retrospective cohort study, our analysis evaluating whether dose reduction/omission is associated with reduced survival is vulnerable to residual confounding.

Our study population was identified from the OCR and is therefore unselected and includes patients of all ages. Our study is considerably larger than previously reported studies and is one of the only studies that is truly population-based. Because of the work required to manually review surgical pathology reports, our study population is a random sample of approximately $25 \%$ of all cases treated in Ontario during the study period. No substantial differences were seen between the randomly selected cases and the cases not included in our study population (see supplemental eTable 1 and eFigure 1, available with this article at JNCCN.org). We did not obtain pathology reports for cases who underwent surgery in 2005. We feel it is very unlikely that this would bias our results in any significant way. Finally, the current study does not address the gap between observed ACT use rates in routine care and optimal treatment rates. This issue is explored in greater detail in an earlier report by our group. ${ }^{21}$ 


\section{Conclusions}

Our results suggest that dose reductions/omissions of adjuvant oxaliplatin for colon cancer are common in routine clinical practice and are more likely in women compared with men. Our data do not show any signal that dose reduction/omission leads to inferior survival. Long-term survival of patients in routine practice is comparable to results observed in clinical trials.

\section{References}

1. NIH consensus conference. Adjuvant therapy for patients with colon and rectal cancer. JAMA 1990;264:1444-1450.

2. IMPACT Investigators. Efficacy of adjuvant fluorouracil and folinic acid in colon cancer. Lancet 1995;345:939-944.

3. Twelves C, Wong A, Nowacki MP, et al. Capecitabine as adjuvant treatment for stage III colon cancer. N Engl J Med 2005;352:2696-2704.

4. Tournigand $\mathrm{C}$, André T, Achille E, et al. FOLFIRI followed by FOLFOX6 or the reverse sequence in advanced colorectal cancer: a randomized GERCOR study. J Clin Oncol 2004;22:229-237.

5. Kuebler JP, Wieand HS, O'Connell MJ, et al. Oxaliplatin combined with weekly bolus fluorouracil and leucovorin as surgical adjuvant chemotherapy for stage II and III colon cancer: results from NSABP C-07. J Clin Oncol 2007;25:2198-2204.

6. Labianca R, Nordlinger B, Beretta GD, et al. Early colon cancer: ESMO Clinical Practice Guidelines for diagnosis, treatment and follow-up. Ann Oncol 2013;24(Suppl 6):vi64-vi72.

7. Benson AB III, Venook AP, Bekjaii-Saab T, et al. NCCN Clinical Practice Guidelines in Oncology: Colon Cancer. Version 2.2016. Accessed August 31,2016 . To view the most recent version of these guidelines, visit NCCN. org.

8. Peppercorn JM, Weeks JC, Cook EF, Joffe S. Comparison of outcomes in cancer patients treated within and outside clinical trials: conceptual framework and structured review. Lancet 2004;363:263-270.

9. Templeton AJ, Vera-Badillo FE, Wang L, et al. Translating clinical trials to clinical practice: outcomes of men with metastatic castration resistant prostate cancer treated with docetaxel and prednisone in and out of clinical trials. Ann Oncol 2013;24:2972-2977.

10. Meyer RM. Generalizing the results of cancer clinical trials. J Clin Oncol 2010;28:187-189.

11. Booth CM, Mackillop WJ. Translating new medical therapies into societal benefit: the role of population-based outcome studies. JAMA 2008;300:2177-2179

12. Booth CM, Tannock IF. Randomised controlled trials and population-based observational research: partners in the evolution of medical evidence. $\mathrm{Br} \mathrm{J}$ Cancer 2014;110:551-555.
13. Murphy CC, Harlan LC, Lund JL, et al. Patterns of colorectal cancer care in the United States: 1990-2010. J Natl Cancer Inst 2015;107:pii: djv198.

14. Sanoff HK, Carpenter WR, Martin CF, et al. Comparative effectiveness of oxaliplatin vs non-oxaliplatin-containing adjuvant chemotherapy for stage III colon cancer. J Natl Cancer Inst 2012;104:211-227.

15. Chagpar R, Xing Y, Chiang YJ, et al. Adherence to stage-specific treatment guidelines for patients with colon cancer. J Clin Oncol 2012;30:972-979.

16. van Gils CW, Koopman M, Mol L, et al. Adjuvant chemotherapy in stage III colon cancer: guideline implementation, patterns of use and outcomes in daily practice in The Netherlands. Acta Oncol 2012;51:57-64.

17. Aspinall SL, Good CB, Zhao X, et al. Adjuvant chemotherapy for stage III colon cancer: relative dose intensity and survival among veterans. BMC Cancer 2015;15:62.

18. Smoragiewicz M, Javaheri KR, Yin Y, Gill S. Neutropenia and relative dose intensity on adjuvant FOLFOX chemotherapy are not associated with survival for resected colon cancer. J Gastrointest Cancer 2014;45:460-465.

19. Sanoff HK, Carpenter WR, Freburger J, et al. Comparison of adverse events during 5-fluorouracil versus 5-fluorouracil/oxaliplatin adjuvant chemotherapy for stage III colon cancer: a population-based analysis. Cancer 2012;118:4309-4320.

20. André T, de Gramont A, Vernerey D, et al. Adjuvant fluorouracil, leucovorin, and oxaliplatin in stage II to III colon cancer: updated 10-year survival and outcomes according to BRAF mutation and mismatch repair status of the MOSAIC study. J Clin Oncol 2015;33:4176-4187.

21. Booth $\mathrm{CM}$, Nanji $\mathrm{S}$, Wei $\mathrm{X}$, et al. Use and effectiveness of adjuvant chemotherapy for stage III colon cancer: a population-based study. J Natl Compr Canc Netw 2016;14:47-56.

22. Clarke EA, Marrett LD, Krieger N. Cancer registration in Ontario: a computer approach. In: Jenson OM, Parkin DM, MacLennan R, eds. Cancer Registration Principles and Methods. Lyon,France: IARC; 1991:246-257.

23. Li X, King C, deGara C, et al. Validation of colorectal cancer surgery data from administrative data sources. BMC Med Res Methodol 2012;12:97.

24. Mackillop WJ, Zhang-Salomons J, Groome PA, et al. Socioeconomic status and cancer survival in Ontario. J Clin Oncol 1997;15:1680-1689.

25. Deyo RA, Cherkin DC, Ciol MA. Adapting a clinical comorbidity index for use with ICD-9-CM administrative databases. J Clin Epidemiol 1992;45:613-619.

26. Yothers G, O'Connell MJ, Allegra CJ, et al. Oxaliplatin as adjuvant therapy for colon cancer: updated results of NSABP C-07 trial, including survival and subset analyses. J Clin Oncol 2011;29:3768-3774.

27. Chansky K, Benedetti J, MacdonaldnJS. Differences in toxicity between men and women treated with 5-fluorouracil therapy for colorectal carcinoma. Cancer 2005;103:1165-1171.

28. Sloan JA, Goldberg RM, Sargent DJ, et al. Women experience greater toxicity with fluorouracil-based chemotherapy for colorectal cancer. J Clin Oncol 2002;20:1491-1498.

29. Kim BH, Bradley T, Tai J, Budman DR. Hypersensitivity to oxaliplatin: an investigation of incidence and risk factors, and literature review. Oncology $2009 ; 76: 231-238$ 